\title{
SUM-PRODUCT ESTIMATES VIA DIRECTED EXPANDERS
}

\author{
VAN H. VU
}

\begin{abstract}
Let $\mathbf{F}_{q}$ be a finite field of order $q$ and $P$ be a polynomial in $\mathbf{F}_{q}\left[x_{1}, x_{2}\right]$. For a set $A \subset \mathbf{F}_{q}$, define $P(A):=\left\{P\left(x_{1}, x_{2}\right) \mid x_{i} \in A\right\}$. Using certain constructions of expanders, we characterize all polynomials $P$ for which the following holds

$$
\text { If }|A+A| \text { is small (compared to }|A| \text { ), then }|P(A)| \text { is large. }
$$
\end{abstract}

The case $P=x_{1} x_{2}$ corresponds to the well-known sum-product problem.

\section{Introduction}

Let $Z$ be a ring and $A$ be a finite subset of $Z$. The sum-product phenomenon, first investigated in [8], can be expressed as follows

$$
\text { If }|A+A| \text { is small, then }|A \cdot A| \text { is large. (*) }
$$

Here "small" and "large" are with respect to $|A|$.

Earlier works on the problem focused on the case $Z$ is $\mathbf{R}$ or $\mathbf{Z}$. In the last few years, starting with [2], the case when $Z$ is a finite field or a modular ring has been studied extensively. This study leads to many important contributions in various areas of mathematics (see [4] for a partial survey).

One of the main applications of sum-product estimates is new constructions of expanders (see, e.g., [3]). In this paper, we investigate the reversed direction and derive sum-product estimates from certain constructions of expanders. In fact, our arguments lead to more general results, described below.

Let $\mathbf{F}_{q}$ be a finite field and $P$ be a polynomial in $\mathbf{F}_{q}\left[x_{1}, x_{2}\right]$. For a set $A \subset \mathbf{F}_{q}$, define $P(A):=\left\{P\left(x_{1}, x_{2}\right) \mid x_{i} \in A\right\}$. As a generalization of $(*)$ (which is the case $P=x_{1} x_{2}$ ), it is tempting to conjecture the following statement

$$
\text { If }|A+A| \text { is small, then }|P(A)| \text { is large. }\left(*^{\prime}\right)
$$

A short consideration reveals, however, that $\left(*^{\prime}\right)$ does not hold for some classes of polynomials. For instance, if $P$ is linear then both $|A+A|$ and $|P(A)|$ can be small at the same time.

Received by the editors May 4, 2007.

The author is supported by NSF Career Grant 0635606. 
Example. Set $P_{1}:=2 x_{1}+3 x_{2}$. Let $A=\{1, \ldots, n\} \subset \mathbf{F}_{q}$, where $q$ is a prime and $1 \leq n \leq q / 10$. Then $|A+A|=2 n-1$ and $\left|P_{1}(A)\right|=5 n-4$.

More generally, if $P$ has the form $P:=Q\left(L\left(x_{1}, x_{2}\right)\right)$ where $Q$ is a polynomial in one variable and $L$ is a linear form, then both $A$ and $|P(A)|$ can be small at the same time.

Example. Set $P_{2}:=\left(2 x_{1}+3 x_{2}\right)^{2}-5\left(2 x_{1}+3 x_{2}\right)+3$. Let $A=\{1, \ldots, n\} \subset \mathbf{F}_{q}$, where $q$ is a prime and $1 \leq n \leq q / 10$. Then $|A+A|=2 n-1$ and $\left|P_{2}(A)\right|=5 n-4$. In this case, $Q=z^{2}-5 z+3$ and $L=P_{1}=2 x_{1}+3 x_{2}$.

Our main result shows that $P:=Q\left(L\left(x_{1}, x_{2}\right)\right)$ is the only (bad) case where the more general phenomenon $(*)^{\prime}$ fails.

Definition 1.1. A polynomial $P \in \mathbf{F}_{q}\left[x_{1}, x_{2}\right]$ is degenerate if it is of the form $Q\left(L\left(x_{1}, x_{2}\right)\right)$ where $Q$ is an one-variable polynomial and $L$ is a linear form in $x_{1}, x_{2}$.

The following refinement of $\left(*^{\prime}\right)$ holds

$$
\text { If }|A+A| \text { is small and } P \text { is non-degenerate, then }|P(A)| \text { is large. (**) }
$$

Theorem 1.2. There is a positive constant $\delta$ such that the following holds. Let $P$ be a non-degenerate polynomial of degree $k$ in $\mathbf{F}_{q}\left[x_{1}, x_{2}\right]$. Then for any $A \subset \mathbf{F}_{q}$

$$
\max \{|A+A|,|P(A)|\} \geq|A| \min \left\{\delta\left(\frac{|A|^{2}}{k^{4} q}\right)^{1 / 4}, \delta\left(\frac{q}{k|A|}\right)^{1 / 3}\right\} .
$$

Remark 1.3. The estimate in Theorem 1.2 is non-trivial when $k^{2} q^{1 / 2} \ll|A| \ll q / k$. In the case when $P$ has fixed degree, this means $q^{1 / 2} \ll|A| \ll q$. This assumption is necessary as if $A$ is a subfield of size $q$ or $q^{1 / 2}$ then $|A+A|=|A|$ and $|P(A)|$ is at most $|A|$. Here and later on $a \ll b$ means $a=o(b)$.

Remark 1.4. Since $P=x_{1} x_{2}$ is clearly non-degenerate, we obtain the following sumproduct estimate, reproving a result from [10]

$$
\max \{|A+A|,|A \cdot A|\} \geq|A| \min \left\{\delta\left(\frac{|A|^{2}}{q}\right)^{1 / 4}, \delta\left(\frac{q}{|A|}\right)^{1 / 3}\right\}
$$

Our arguments can be extended to modular rings. Let $m$ be a large integer and $\mathbf{Z}_{m}$ be the ring consisting of residues mod $m$. Let $\gamma(m)$ be the smallest prime divisor of $m$ and $\tau(m)$ be the number of divisors of $m$. Define $g(m):=\sum_{n \mid m} \tau(m) \tau(m / n)$.

Theorem 1.5. There is a positive constant $\delta$ such that the following holds. Let $A$ be a subset of $\mathbf{Z}_{m}$. Then

$$
\max \{|A+A|,|A \cdot A|\} \geq|A| \min \left\{\delta \frac{\gamma(m)^{1 / 4}|A|^{1 / 2}}{g(m)^{1 / 2} m^{1 / 2}}, \delta\left(\frac{m}{|A|}\right)^{1 / 3}\right\} .
$$


Remark 1.6. This theorem is effective when $m$ is the product of few large primes.

Our study was motivated by two papers [14] and [10]. In these papers, the authors used an argument based on Kloosterman sums estimates to study Cayley graphs and the sum-product problem, respectively. Our approach here relies on a combination of a generalization of this argument and the spectral method from graph theory.

\section{Erdös' distinct distances problem}

The following question, asked by Erdős in the 1940's [7], is among the most well known problems in discrete geometry

Question 2.1. What is the minimum number of distinct distances (in euclidean norm) determined by $n$ points on the plane?

For a point set $A$, we denote by $\Delta(A)$ the set of distinct distances in $A$. It is easy to show that $|\Delta(A)|=\Omega\left(|A|^{1 / 2}\right)$. To see this, consider an arbitrary point $a \in A$. If from $a$ there are $|A|^{1 / 2}$ different distances, then we are done. Otherwise, by the pigeon hole principle, there is a circle centered at $a$ containing at least $|A|^{1 / 2}$ other points. Take a point $a^{\prime}$ on this circle. Since two circles intersect in at most 2 points, there are at least $\frac{|A|^{1 / 2}-1}{2}$ distinct distances from $a^{\prime}$ to the other points on the circle.

It has been conjectured that $|\Delta(A)| \geq|A|^{1-o(1)}$ (the $o(1)$ term is necessary as shown by the square grid). This conjecture is still open. For the state of the art of this problem, we refer to $[15$, Chapter 6$]$.

What happens if one replaces the euclidean distance by other distances ? One can easily see that for the $l_{1}$ distance, the conjectured bound $|\Delta(A)| \geq|A|^{1-o(1)}$ fails, as the square grid determines only $|A|^{1 / 2}$ distances. On the other hand, it seems reasonable to think that there is no essential difference between the $l_{2}$ and (say) the $l_{4}$ norms. In fact, in [9], it was shown that certain arguments used to handle the $l_{2}$ case can be used, with some more care, to handle a wide class of other distances.

The finite field version of Erdős problem was first considered in [2], with the euclidean distance (see also [11] for more recent development). Here we extend this work for a general distance. Let $P$ be a symmetric polynomial in two variables. (By symmetry, we mean that $P$ is symmetric around the origin, i.e., $P(x, y)=P(-x,-y)$.) Define the $P$-distance between two points $x=\left(x_{1}, x_{2}\right)$ and $y=\left(y_{1}, y_{2}\right)$ in the finite plane $\mathbf{F}_{q}^{2}$ as $P\left(y_{1}-x_{1}, y_{2}-x_{2}\right)$. Let $\Delta_{P}(A)$ be the set of distinct $P$-distances in $A$.

Theorem 2.2. There is a positive constant $\delta$ such that the following holds. Let $P$ be a symmetric non-degenerate polynomial of degree $k$ and $A$ be a subset of the finite plane $\mathbf{F}_{q}^{2}$, then

$$
\left|\Delta_{P}(A)\right| \geq \delta \min \left\{\frac{|A|}{k^{2} \sqrt{q}}, \frac{q}{k}\right\}
$$


Remark 2.3. The polynomial $P=x^{p}+y^{p}$, which corresponds to the $l_{p}$ norm, is non-degenerate for any positive integer $p \geq 2$.

Remark 2.4. Assume that $k=O(1)$. For $|A| \gg q$, the term $\frac{|A|}{\sqrt{q}} \gg|A|^{1 / 2}$, and so $\left|\Delta_{P}(A)\right| \gg|A|^{1 / 2}$. If $A \mid \leq q$, one cannot expect a bound better than $|A|^{1 / 2}$, as $A$ can be a sub-plane.

Remark 2.5. The proof also works for a non-symmetric $P$. In this case, $\operatorname{dist}(x, y)$ and $\operatorname{dist}(y, x)$ may be different.

\section{Directed expanders and spectral gaps}

Let $G$ be a $d$-regular graph on $n$ vertices and $A_{G}$ be the adjacency matrix of $G$. The rows and columns of $A_{G}$ are indexed by the vertices of $G$ and the entry $a_{i j}=1$ if $i$ is adjacent to $j$ in $G$ and zero otherwise. Let $d=\lambda_{1}(G) \geq \lambda_{2}(G) \geq \cdots \geq \lambda_{n}(G)$ be the eigenvalues of $A_{G}$. Define

$$
\lambda(G):=\max \left\{\left|\lambda_{2}\right|,\left|\lambda_{n}\right|\right\} .
$$

It is well known that if $\lambda(G)$ is significantly less than $d$, then $G$ behaves like a random graphs (see, for example, [6] or [1]). In particular, for any two vertex sets $B$ and $C$

$$
\left|e(B, C)-\frac{d}{n}\right| B|| C|| \leq \lambda(G) \sqrt{|B||C|} .
$$

where $e(B, C)$ is the number of edges with one end point in $B$ and the other in $C$.

We are going to develop a directed version of this statement. Let $G$ be a directed graph (digraph) on $n$ points where the out-degree of each vertex is $d$. The adjacency matrix $A_{G}$ is defined as follows: $a_{i j}=1$ if there is a directed edge from $i$ to $j$ and zero otherwise. Let $d=\lambda_{1}(G), \lambda_{2}(G), \ldots, \lambda_{n}(G)$ be the eigenvalues of $A_{G}$. (These numbers can be complex so we cannot order them, but by Frobenius' theorem all $\left|\lambda_{i}\right| \leq d$.) Define

$$
\lambda(G):=\max _{i \geq 2}\left|\lambda_{i}\right|
$$

An $n$ by $n$ matrix $A$ is normal if $A^{\top} A=A A^{\top}$. We say that a digraph is normal if its adjacency matrix is a normal matrix. There is a simple way to test whether a digraph is normal. In a digraph $G$, let $N^{+}(x, y)$ be the set of vertices $z$ such that both $x z$ and $y z$ are (directed) edges. Similarly, let $N^{-}(x, y)$ be the set of vertices $z$ such that both $z x$ and $z y$ are (directed) edges. It is easy to see that $G$ is normal if and only if 


$$
\left|N^{+}(x, y)\right|=\left|N^{-}(x, y)\right|
$$

for any two vertices $x$ and $y$.

Lemma 3.1. Let $G$ be a normal directed graph on $n$ vertices with all out-degree equal d. Let $d=\lambda_{1}(G), \lambda_{2}(G), \ldots, \lambda_{n}(G)$ be the eigenvalues of $A_{G}$. Then for any two vertex sets $B$ and $C$

$$
\left|e(B, C)-\frac{d}{n}\right| B|| C|| \leq \lambda(G) \sqrt{|B||C|}
$$

where $e(B, C)$ is the number of (directed) edges from $B$ to $C$.

Proof. The eigenvector of $\lambda_{1}=d$ is $\mathbf{1}$, the all-one vector. Let $v_{i}, 2 \leq i \leq n$, be the eigenvectors of $\lambda_{i}$. A well known fact from linear algebra asserts that if $A$ is normal then its eigenvectors form an orthogonal basis of $\mathbf{K}^{n}$ (where $\mathbf{K}$ denotes the field of complex numbers). It follows that any vector $x$ orthogonal to $\mathbf{1}$ can be written as a linear combination of these $v_{i}$. By the definition of $\lambda$ we have that for any such vector $x$

$$
\left\|A_{G} x\right\|^{2}=<A_{G} x, A_{G} x>\leq \lambda^{2}\|x\|^{2} .
$$

From here one can use the same arguments as in the non-directed case to conclude the proof. We reproduce these arguments (from [1]) for the reader's convenience.

Let $V:=\{1, \ldots, n\}$ be the vertex set of $G$. Set $c:=|C| / n$ and let $x:=\left(x_{1}, \ldots, x_{n}\right)$ where $x_{i}:=I_{i \in C}-c$. It is clear that $x$ is orthogonal to 1 . Thus,

$$
<A x, A x>\leq \lambda(G)^{2}\|x\|^{2}
$$

The right hand side is $\lambda_{G}^{2} c(1-c) n \leq \lambda_{G}^{2} c n=\lambda(G)^{2}|C|$. The left hand side is $\sum_{v \in V}\left(\left|N_{C}(v)\right|-c d\right)^{2}$, where $N_{C}(v)$ is the set of $v^{\prime} \in C$ such that $v v^{\prime}$ is an directed edge. It follows that

$$
\sum_{v \in B}\left(\mid N_{C}(v)-c d\right)^{2} \leq \sum_{v \in V}\left(\left|N_{C}(v)\right|-c d\right)^{2} \leq \lambda^{2}|C|
$$

On the other hand, by the triangle inequality 


$$
\left|e(B, C)-\frac{d}{n}\right| B|| C||=|e(B, C)-c d| B|| \leq \sum_{v \in B}\left|N_{C}(v)-c d\right| .
$$

By Cauchy-Schwartz and (2), the right hand side of (3) is bounded from above by

$$
\sqrt{|B|}\left(\sum_{v \in B}\left(N_{C}(v)-c d\right)^{2}\right)^{1 / 2} \leq \lambda \sqrt{|B||C|}
$$

concluding the proof.

Now we are ready to formalize our first main lemma:

Lemma 3.2. (Expander decomposition lemma) Let $\overrightarrow{K_{n}}$ be the complete digraph on $V:=\{1, \ldots, n\}$. Assume that $\overrightarrow{K_{n}}$ is decomposed in to $k+1$ edge-disjoint digraphs $H_{0}, H_{1}, \ldots, H_{k}$ such that

- For each $i=1, \ldots, k$, the out-degrees in $H_{i}$ are the same and at most $d$ and $\lambda\left(H_{i}\right) \leq \lambda$.

- The out-degrees in $H_{0}$ are at most $d^{\prime}$.

Let $B$ and $C$ be subsets of $V$ and $K$ be a subgraph of $\overrightarrow{K_{n}}$ with $L$ (directed) edges going from $B$ to $C$. Then $K$ contains edges from at least

$$
\min \left\{\frac{L-|B| d^{\prime}}{2 \lambda \sqrt{|B||C|}}, \frac{\left(L-|B| d^{\prime}\right) n}{2 d|B||C|}\right\}
$$

different $H_{i}, i \geq 1$.

Proof. By the previous lemma, each $H_{i}, 1 \leq i \leq k$ has at most

$$
\frac{d}{n}|B||C|+\lambda \sqrt{|B||C|}
$$

edges going from $B$ to $C$. Furthermore, $H_{0}$ has at most $|B| d^{\prime}$ edges going from $B$ to $C$. Thus the number of $H_{i}, i \geq 1$, having edges in $K$ is at least

$$
\left(\frac{d}{n}|B||C|+\lambda \sqrt{|B||C|}\right)^{-1}\left(L-d^{\prime}|B|\right) \geq \min \left\{\frac{L-d^{\prime}|B|}{2 \lambda \sqrt{|B||C|}}, \frac{\left(L-d^{\prime}|B|\right) n}{2 d|B||C|}\right\}
$$

completing the proof. 


\section{Directed Cayley graphs}

Let $H$ be a finite (additive) abelian group and $S$ be a subset of $H$. Define a directed graph $G_{S}$ as follows. The vertex set of $G$ is $H$. There is a direct edge from $x$ to $y$ if and only if $y-x \in S$. It is clear that every vertex in $G_{S}$ has out-degree $|S|$. (In general $H$ can be non-abelian, but in this paper we restrict ourselves to this case.)

Let $\chi_{\xi}, \xi \in H$, be the (additive) characters of $H$. It is well known that for any $\xi \in H$, $\sum_{s \in S} \chi_{\xi}(s)$ is an eigenvalue of $G_{S}$, with respect the eigenvector $\left(\chi_{\xi}(x)\right)_{x \in H}$.

It is important to notice that the graph $G_{S}$, for any $S$, is normal, by (1). Indeed, for any two vertex $x$ and $y$

$$
\left|N^{+}(x, y)\right|=\left|N^{-}(x, y)\right|=|(x+S) \cap(y+S)| .
$$

We are going to focus on the following two cases

Special case 1. $H=\mathbf{F}_{q}^{2}$, with $\mathbf{F}_{q}$ being a finite field of $q=p^{r}$ elements, $p$ prime. Using $e(\alpha)$ to denote $\exp \left(\frac{2 \pi i}{p} \alpha\right)$, we have

$$
\chi_{\xi}(x)=\exp \left(\frac{2 \pi i}{p} \operatorname{Trace} \xi \cdot x\right)=e(\operatorname{Trace} \xi \cdot x)
$$

where Trace $z:=z+z^{p}+\cdots+z^{p^{r-1}}$ and $\xi \cdot x$ is the inner product of $\xi$ and $x$.

Special case 2. $H=\mathbf{Z}_{m}^{2}$. In this case we use $e(\alpha)$ to denote $\exp \left(\frac{2 \pi i}{m} \alpha\right)$. We have

$$
\chi_{\xi}(x)=\exp \left(\frac{2 \pi i}{m} \xi \cdot x\right)=e(\xi \cdot x)
$$

Our second main ingredient is the following theorem, which is a corollary of [12, Theorem 5.1.1]. (We would like to thank B. C. Ngo for pointing out this reference.)

Theorem 4.1. Let $P$ be a polynomial of degree $k$ in $\mathbf{F}_{q}\left[x_{1}, x_{2}\right]$ which does not contain a linear factor. Let $\operatorname{Root}(\mathrm{P})$ be the set of roots of $P$ in $\mathbf{F}_{q}^{2}$. Then for any $0 \neq y \in \mathbf{F}_{q}^{2}$,

$$
\left|\sum_{x \in \operatorname{Root}(\mathrm{P})} e(x \cdot y)\right|=O\left(k^{2} q^{1 / 2}\right)
$$

Given a polynomial $P$ and an element $a \in \mathbf{F}_{q}$, we denote by $G_{a}$ the Cayley graph defined by the set $\operatorname{Root}(\mathrm{P}-\mathrm{a})$. As a corollary of the theorem above, we have

Corollary 4.2. Let $P$ be a polynomial of degree $k$ in $\mathbf{F}_{q}\left[x_{1}, x_{2}\right]$ and a be an element of $F_{q}$ such that $P-a$ does not contain a linear factor. Then $\lambda\left(G_{a}\right)=O\left(k^{2} q^{1 / 2}\right)$. 
It is plausible that a ring analogue of Theorem 4.1 can be derived (with $\mathbf{F}_{q}$ replaced by $\mathbf{Z}_{m}$ ). However, the (algebraic) machinery involved is heavy. We shall give a direct proof for Corollary 4.2 in the special case when $P$ is quadratic.

Let $\Omega$ be the set of those quadratic polynomials which (after a proper changing of variables) can be written in the form $A_{1} x^{2}+A_{2} y^{2}$ with $A_{1}, A_{2} \in \mathbf{Z}_{m}^{*}$, the set of elements co-prime with $m$. (For example, both $Q=x^{2}+y^{2}$ and $Q=2 x y=$ $(x+y)^{2}-(x-y)^{2}$ belong to $\Omega$.) Fix a $Q$ in $\Omega$ and for each $a \in \mathbf{Z}_{m}$ define the Cayley graph $G_{a}$ as before.

Theorem 4.3. For any $0 \neq a \in \mathbf{Z}_{m}$,

$$
\lambda\left(G_{a}\right) \leq g(m) \frac{m}{\gamma(m)^{1 / 2}}
$$

The proof of this theorem will appear in Section 6 .

\section{Proofs of Theorems 1.2 and 1.5}

To prove Theorem 1.2, consider a set $A \subset \mathbf{F}_{q}$ and set $B:=A \oplus A \subset \mathbf{F}_{q}^{2}$. Since our estimate is trivial if $|A|=O\left(k^{2} q^{1 / 2}\right)$, we assume that $|A| \gg k^{2} q^{1 / 2}$.

For each $a \in \mathbf{F}_{q}$, consider the polynomial $P_{a}=P-a$ and define a Cayley graph $G_{a}$ accordingly. The out-degree in this graph is $O(q)$. We say that an element $a$ is good if $P-a$ does not contain a linear factor and bad other wise.

Lemma 5.1. Let $P$ be a polynomial of degree $k$ in $\mathbf{F}_{q}\left[x_{1}, \ldots, x_{d}\right]$. Assume that $P$ cannot be written in the form $P=Q(L)$, where $Q$ a polynomial with one variable and $L$ is a linear form of $x_{1}, \ldots, x_{d}$. Then there are at most $k-1$ elements $a_{i}$ such that the polynomial $P-a_{i}$ contains a linear factor.

Proof. Let $a_{1}, \ldots, a_{k}$ be different elements of $\mathbf{F}_{q}$ such that there are linear forms $L_{1}, \ldots, L_{k}$ and polynomials $P_{1}, \ldots, P_{k} \in \mathbf{F}_{q}\left[x_{1}, \ldots, x_{d}\right]$ such that $P-a_{i}=L_{i} P_{i}$.

If $L_{i}$ and $L_{j}$ had a common root $x$, then $P(x)-a_{i}=P(x)-a_{j}=0$, a contradiction as $a_{i} \neq a_{j}$. It follows that for any $1 \leq i<j \leq d, L-i$ and $L_{j}$ do not have a common root. But since the $L_{i}$ are linear forms, we can conclude that they are translates of the same linear form $L$, i.e., $L_{i}=L-b_{i}$, for some $b_{1}, \ldots, b_{k} \in \mathbf{F}_{q}$.

It now suffices to prove the following claim

Lemma 5.2. Let $P$ be a polynomial in $\mathbf{F}_{q}\left[x_{1}, \ldots, x_{d}\right]$ of degree $k$. Assume that there is a non-zero linear form $L$, a sequence $a_{1}, \ldots, a_{k}$ of (not necessarily distinct) elements of $\mathbf{F}_{q}$ and a set $\left\{b_{1}, \ldots, b_{k}\right\} \subset \mathbf{F}_{q}$ such that $P(x)=a_{i}$ whenever $L(x)=b_{i}$. Then there is a polynomial $Q$ in one variable such that $P=Q(L)$. 
Assume, without loss of generality, that the coefficient of $x_{1}$ in $L$ is non-zero. We are going to induct on the degree of $x_{1}$ in $P$ (which is at most $k$ ). If this degree is 0 (in other words $P$ does not depend on $x_{1}$ ), then $P$ is a constant, since for any sequence $x_{2}, \ldots, x_{d}$, we can choose an $x_{1}$ such that $L\left(x_{1}, \ldots, x_{d}\right)=b_{1}$, so

$$
P\left(x_{1}, \ldots, x_{d}\right)=a_{1} .
$$

If the degree in concern is not zero, then we can write

$$
P=\left(L-b_{1}\right) P_{1}^{\prime}(x)+Q_{1}
$$

where $Q_{1}$ does not contain $x_{1}$. By the above argument, we can show that $Q_{1}=a_{1}$. Furthermore, if $L(x)=b_{i}, 2 \leq i \leq k$, then $P_{1}^{\prime}=\left(a_{i}-a_{1}\right) /\left(b_{i}-b_{1}\right)$. Now apply the induction hypothesis on $P_{1}^{\prime}$, whose $x_{1}$-degree is one less than that of $P_{1}$.

If $a$ is good, then $\lambda\left(G_{a}\right)=O\left(k^{2} q^{1 / 2}\right)$. Let the graph $H_{0}$ be the union of bad $G_{a}$. By the above lemma, the maximum out-degree of this graph is $d^{\prime}=O\left(k^{2} q\right)$.

In $\mathbf{F}_{q}^{2}$, define a directed graph $K$ by drawing a directed edge from $(x, y)$ to $\left(x^{\prime}, y^{\prime}\right)$ if and only if either both $x^{\prime}-x$ and $y^{\prime}-y$ are in $A$ or both $x-x^{\prime}$ and $y-y^{\prime}$ are in $A$. Consider the set $C:=(A+A) \oplus(A+A) \subset \mathbf{F}_{q}^{2}$. Notice that in $K$ any point from $B$ has at least $|A|^{2}$ edges going into $C$. Thus $L$, the number of directed edges from $B$ to $C$, is at least $|A|^{4}$. Since $|A| \geq k^{2} q^{1 / 2}$, we have

$$
L-|B| d^{\prime} \geq|A|^{4}-|B| d^{\prime}=|A|^{4}-|A|^{2} O\left(k^{2} q\right)=(1-o(1))|A|^{4} .
$$

Applying the Expander Decomposition Lemma and Corollary 4.2, we can conclude that the number of $P_{a}$ having edges from $B$ to $C$ (which, by definition of $B$ and $C$, is $|P(A)|)$, is at least

$$
\Omega\left(\min \left\{(1-o(1)) \frac{|A|^{4}}{k^{2} q^{1 / 2}|A||A+A|}, \frac{(1-o(1))|A|^{4} q^{2}}{k q|A|^{2}|A+A|^{2}}\right\}\right) .
$$

from which the desired estimate follows by Hölder inequality. The proof of Theorem 1.5 (using Theorem 4.3 instead of Corollary 4.2) is similar and is left as an exercise.

To prove Theorem 2.2, consider a set $A \subset \mathbf{F}_{q}^{2}$ where $|A| \gg k q$. Let $B=C=A$ and $K$ be the complete digraph on $A$. We can assume that $\mid A \gg q$. We have $L=(1+o(1))|A|^{2}$ and $d^{\prime}=O(k q)$. Thus $L-d^{\prime}|B|=L-d^{\prime}|A|=(1+o(1)) L=$ $(1+o(1))|A|^{2}$. By the Expander Decomposition Lemma, 


$$
\left|\Delta_{P}(A)\right|=\Omega\left(\min \left\{(1-o(1)) \frac{|A|^{2}}{k^{2} q^{1 / 2}|A|}, \frac{(1-o(1))|A|^{2} q^{2}}{k q|A|^{2}}\right\}\right) .
$$

The right hand side is

completing the proof.

$$
\Omega\left(\min \left\{\frac{|A|}{k^{2} q^{1 / 2}}, \frac{q}{k}\right\}\right)
$$

\section{Proof of Theorem 4.3}

We are going to follow an approach from [14]. We need to use the following two classical estimates (see, for example, [13, page 19])

Theorem 6.1. (Gauss sum) Let $m$ be an positive odd integer. Then for any integer $z$ co-prime to $m$

$$
\left|\sum_{y \in \mathbf{Z}_{m}} e\left(z y^{2}\right)\right|=\sqrt{m}
$$

Theorem 6.2. (Kloosterman sum) Let $m$ be an positive odd integer. Then

$$
\left|\sum_{y \in \mathbf{Z}_{m}^{*}} e(a y+b \bar{y})\right| \leq \tau(m)(a, b, m)^{1 / 2} \sqrt{m}
$$

where $(a, b, m)$ is the greatest common divisor of $a, b$ and $m, \tau(m)$ is the number of divisors of $m$, and $\bar{y}$ is the inverse of $y$.

Let $p_{1}<\cdots<p_{k}$ be the prime divisors of $m$ and set $\Omega(m):=\left\{\prod_{i \in I} p_{i} \mid I \subset\right.$ $\{1, \ldots, k\}, I \neq \emptyset\}$. Notice that $g(m)$ satisfies the following recursive formula: $g(1):=$ 0 and $g(m):=\tau(m)+\sum_{d \in \Omega(m)} g(m / d)$.

Let $S$ be the set of roots of $Q-a$. We are going to use the notation $G_{S}$ instead of $G_{a}$.

We use induction on $m$ to show that

$$
\left|\lambda\left(G_{S}\right)\right| \leq g(m) \frac{m}{\gamma(m)^{1 / 2}}
$$

The case $m=1$ is trivial, so from now on we assume $m>1$. By properties of Cayley's graphs, the eigenvalues of $G_{S}$ are 


$$
\lambda_{\xi}=\sum_{s \in S} e(\xi \cdot s)
$$

where $\xi \in \mathbf{Z}_{m}^{2}$. For $\xi=0$, we obtain the largest eigenvalue $|S|$, which is the degree of the graph. In what follows, we assume that $\xi \neq 0$. Recall that $s \in S$ if and only if $Q(s)=a$. We have

$$
m \lambda_{\xi}=\sum_{x \in \mathbf{Z}_{m}^{2}} \sum_{v \in \mathbf{Z}_{m}} e(-a v) e(\xi \cdot x+v Q(x))=\sum_{v \in \mathbf{Z}_{m} \backslash\{0\}} F(v)
$$

where $F(v):=\sum_{x \in \mathbf{Z}_{m}^{2}} e(-a v) e(\xi \cdot x+v Q(x))$, taking into account the fact that $F(0)=0$.

For $d=\prod_{i \in I} p_{i} \in \Omega(m)$, let $\eta(d)=|I|+1$. By the exclusion-inclusion formula,

$$
\sum_{v \in \mathbf{Z}_{m} \backslash 0} F(v)=\sum_{v \in \mathbf{Z}_{m}^{*}} F(v)+\sum_{d \in \Omega(m)}(-1)^{\eta(d)} \sum_{d \mid v} F(v) .
$$

Let us first bound $S_{0}:=\sum_{v \in \mathbf{Z}_{m}^{*}} F(v)$. We write $x=\left(x_{1}, x_{2}\right)$ where $x_{1}, x_{2} \in \mathbf{Z}_{m}$. As $Q$ is non-degenerate, by changing variables we can rewrite $e(\xi \cdot x+v Q(x))$ as $e\left(v\left(A_{1} x_{1}^{2}+A_{2} x_{2}^{2}\right)+\left(B_{1} x_{1}+B_{2} x_{2}+C\right)\right)$ where $B_{1}, B_{2}, C$ may depend on $\xi$, but $A_{1}, A_{2} \in \mathbf{Z}_{m}^{*}$ depends only on $Q$. We have (thanks to the fact that $v, A_{1}, A_{2}, 2,4$ are all in $\mathbf{Z}_{m}^{*}$ )

$$
\begin{aligned}
& v\left(A_{1} x_{1}^{2}+A_{2} x_{2}^{2}\right)+\left(B_{1} x_{1}+B_{2} x_{2}+C\right) \\
= & v A_{1}\left(x_{1}+\frac{B_{1}}{2 v A_{1}}\right)^{2}+v A_{2}\left(x_{2}+\frac{B_{2}}{2 v A_{2}}\right)^{2}+\left(C-\frac{B_{1}^{2}}{4 v A_{1}}-\frac{B_{2}^{2}}{4 v A_{2}}\right) .
\end{aligned}
$$

It follows that

$$
\begin{aligned}
S_{0}= & \sum_{v \in \mathbf{Z}_{m}^{*}} e(C) e\left(-a v-\left(\frac{B_{1}^{2}}{4 A_{1}}+\frac{B_{2}^{2}}{4 A_{2}}\right) \bar{v}\right) \\
& \times \sum_{x_{1}, x_{2} \in \mathbf{Z}_{m}} e\left(v A_{1}\left(x_{1}+\frac{B_{1}}{2 v A_{1}}\right)^{2}+v A_{2}\left(x_{2}+\frac{B_{2}}{2 v A_{2}}\right)^{2}\right) .
\end{aligned}
$$

Notice that 
(7)

$$
\sum_{x_{1}, x_{2} \in \mathbf{Z}_{m}} e\left(v A_{1}\left(x_{1}+\frac{B_{1}}{2 v A_{1}}\right)^{2}+v A_{2}\left(x_{2}+\frac{B_{2}}{2 v A_{2}}\right)^{2}\right)=\sum_{y \in \mathbf{Z}_{m}} e\left(v A_{1} y^{2}\right) \sum_{y \in \mathbf{Z}_{m}} e\left(v A_{2} y^{2}\right) .
$$

Set $b:=\frac{B_{1}^{2}}{4 v A_{1}}+\frac{B_{2}^{2}}{4 v A_{2}}$, we have

$$
S_{0}=e(C)\left(\sum_{y \in \mathbf{Z}_{m}} e\left(v A_{1} y^{2}\right) \sum_{y \in \mathbf{Z}_{m}} e\left(v A_{2} y^{2}\right)\right) \sum_{v \in \mathbf{Z}_{m}^{*}} e(-a v-b \bar{v}) .
$$

By Theorems 6.1 and 6.2 and the fact that $(a, b, m) \leq \frac{m}{\gamma(m)}$ (since $\left.a \neq 0\right)$, we have

$$
\left|S_{0}\right| \leq m \tau(m)(a, b, m)^{1 / 2} m^{1 / 2} \leq \tau(m) \frac{m^{2}}{\gamma(m)^{1 / 2}}
$$

Now we bound the second term in the right hand side of (5), using the induction hypothesis. Fix $d \in \Omega(m)$ and consider

$$
S_{d}:=\sum_{d \mid v} F(v)=\sum_{x \in \mathbf{Z}_{m}^{2}} e(\xi \cdot x) \sum_{d \mid v} e(v(Q(x)-a)) .
$$

Write $m=d m_{d}, v=d v^{\prime}$, where $m_{d}:=m / d$ and $v^{\prime} \in \mathbf{Z}_{m_{d}}$. Each vector $x$ in $\mathbf{Z}_{m}^{2}$ has a unique decomposition $x=x^{[1]}+m_{d} x^{[2]}$ where $x^{[1]} \in \mathbf{Z}_{m_{d}}^{2}$ and $x^{[2]} \in \mathbf{Z}_{d}^{2}$. Finally, there is $a^{\prime} \in \mathbf{Z}_{m_{d}}$ such that $a \equiv a^{\prime}\left(\bmod m_{d}\right)$. Since $Q(x) \equiv Q\left(x^{[1]}\right)\left(\bmod m_{d}\right)$, we have

$$
e(v(Q(x)-a))=\exp \left(\frac{2 \pi i}{m_{d}} v^{\prime}\left(Q\left(x^{\left[x_{1}\right]}-a^{\prime}\right)\right) .\right.
$$

Therefore,

$$
\sum_{d \mid v} e(v(Q(x)-a))=\sum_{v^{\prime} \in \mathbf{Z}_{m_{d}}} \exp \left(\frac { 2 \pi i } { m _ { d } } v ^ { \prime } \left(Q\left(x^{[1]}-a^{\prime}\right)\right.\right.
$$

which equals $m_{d}$ if $Q\left(x^{[1]}\right) \equiv a^{\prime}\left(\bmod m_{d}\right)$ and zero otherwise. It follows that 


$$
S_{d}=m_{d} \sum_{x \in \mathbf{Z}_{m}^{2}, Q\left(x^{[1]}\right)=a^{\prime}\left(\bmod m_{d}\right)} e(\xi \cdot x) .
$$

Next, we rewrite $e(\xi \cdot x)$ as $\exp \left(\frac{2 \pi i}{m}\left(\xi \cdot x^{[1]}+m_{d} \xi \cdot x^{[2]}\right)\right)$. This way, we have

$$
S_{d}=m_{d} \sum_{x^{[1]} \in \mathbf{Z}_{m_{d}}^{2}, Q\left(x^{[1]}\right)=a^{\prime}} e\left(\xi \cdot x^{[1]}\right) \sum_{x^{[2]} \in \mathbf{Z}_{d}^{2}} \exp \left(\frac{2 \pi i}{d} \xi \cdot x_{2}\right) .
$$

The sum $\sum_{x^{[2]} \in \mathbf{Z}_{d}^{2}} \exp \left(\frac{2 \pi i}{d} \xi \cdot x_{2}\right)$ is $d^{2}$ if both coordinates of $\xi$ are divisible by $d$ and zero other wise. Set $\xi_{d}=\xi / d$, we have

$$
S_{d}=m_{d} d^{2} \sum_{Q\left(x^{[1]}\right)=a^{\prime}} \exp \left(\frac{2 \pi i}{m_{d}} \xi_{d} \cdot x^{[1]}\right)
$$

Notice that $\sum_{Q\left(x^{[1]}\right)=a^{\prime}} \exp \left(\frac{2 \pi i}{m_{d}} \xi_{d} \cdot x^{[1]}\right.$ is a (non-trivial) eigenvalue of a Cayley's graph defined by $Q$ on $\mathbf{Z}_{m_{d}}^{2}$, where $m_{d}=m / d$. Thus, by the induction hypothesis,

$$
\left|\sum_{Q\left(x^{[1]}\right)=a^{\prime}} \exp \left(\frac{2 \pi i}{m_{d}} \xi_{d} \cdot x^{[1]}\right)\right| \leq g(m / d) \frac{m / d}{\gamma(m / d)^{1 / 2}} \leq g(m / d) \frac{m / d}{\gamma(m)^{1 / 2}}
$$

This implies

$$
\left|S_{d}\right| \leq g(m / d) \frac{m^{2}}{\gamma(m)^{1 / 2}}
$$

By (5), (9), (12) and the triangle inequality

$$
m \lambda_{\xi} \leq \frac{m^{2}}{\gamma(m)^{1 / 2}}\left(\tau(m)+\sum_{d \in \Omega(m)} g(m / d)\right)=g(m) \frac{m^{2}}{\gamma(m)^{1 / 2}}
$$

completing the proof.

\section{Open questions}

Our study leads to several questions: 
Problem 1. What happens if $|A| \leq q^{1 / 2}$ in the case $q$ is a prime?

Problem 2. If $q$ is not a prime and both $|A+A|$ and $|P(A)|$ is small, can one prove that most of $A$ is contained in a subfield ?

Problem 3. Characterize all pairs $P_{1}, P_{2}$ of polynomials such that the following generalization of $(*)$ holds:

$$
\text { If }\left|P_{1}(A)\right| \text { is small, then }\left|P_{2}(A)\right| \text { is large. }
$$

Problem 4. Assume that $q$ is a prime. Let $\epsilon$ be a small positive constant. A polynomial in $\mathbf{F}_{q}\left[x_{1}, x_{2}\right]$ is generic if for any sufficiently large $A \subset \mathbf{F}_{q},|P(A)| \geq$ $\min \left\{q,|A|^{1+\epsilon}\right\}$. Can one characterize all generic polynomials ?

\section{References}

[1] N. Alon, J. Spencer, The probabilistic method (Second edition), Wiley-Interscience, 2000.

[2] J. Bourgain, N. Katz, and T. Tao, A sum-product estimate in finite fields, and applications, Geom. Funct. Anal. 14 (2004), no. 1, 27-57.

[3] J. Bourgain and A. Gamburd, New results on expanders, C. R. Math. Acad. Sci. Paris, 342 (2006), no. 10, 717-721.

[4] J. Bourgain, More on the sum-product phenomenon in prime fields and its applications, Int. J. Number Theory, 1 (2005), no. 1, 1-32.

[5] P. Brass, W. Moser, and J. Pach, Research problems in discrete geometry, Springer, New York, 2005.

[6] F. Chung, R. Graham, and R. Wilson, Quasi-random graphs, Combinatorica 9 (1989), no. 4, 345-362.

[7] P. Erdős, On sets of distances of $n$ points, Amer. Math. Monthly, 53 (1946), 248-250.

[8] P. Erdős and E. Szemerédi, On sums and products of integers, Studies in pure mathematics, 213-218, Birkhuser, Basel, 1983.

[9] J. Garibaldi, Erdős Distance Problem for Convex Metrics, Ph. D. Thesis, UCLA 2004.

[10] D. Hart, A. Iosevich, and J. Solymosi, Sum-product estimates in finite fields via Kloosterman sums, Int. Math. Res. Not. IMRN 2007, no. 5, Art. ID rnm007, 14 pp.

[11] A. Iosevich and M. Rudnev, Erdös distance problem in vector spaces over finite fields, Trans. Amer. Math. Soc. 359 (2007), no. 12, 6127-6142.

[12] N. Katz, Sommes exponentielles, Asterisque 79, Socit Mathmatique de France, Paris, 1980.

[13] H. Iwaniec and E. Kowalski, Analytic number theory, American Mathematical Society, 2004.

[14] A. Medrano, P. Myers, H. M. Stark, and A. Terras, Finite analogues of Euclidean space, J. Comput. Appl. Math. 68 (1996), no. 1-2, 221-238.

[15] T. Tao and V. Vu, Additive combinatorics, Cambridge University Press, 2006.

Department of Mathematics, Rutgers, Piscataway, NJ 08854

E-mail address: vanvu@@math.rutgers.edu 